\title{
Oscillation of a pendulum subject to a horizontal trajectory with different kinds of motion
}

\section{Oscilación de un péndulo sujeto a una trayectoria horizontal con diferentes clases de movimiento}

\author{
ESPINDOLA-HEREDIA, Rodolfo $\dagger^{*}$, DEL VALLE, Gabriela, MUCIÑO-CRUZ, Damián and \\ HERNANDEZ-MORALES, Guadalupe
}

Universidad Autónoma Metropolitana-Unidad Azcapotzalco, División de Ciencias Básicas e Ingeniería, Dpto. Ciencias Básicas, área Física Atómica Molecular Aplicada, Laboratorio de Investigación en Dinámica Rotacional, Edificio G-103.

ID $1^{\text {st }}$ Author: Rodolfo, Espindola-Heredia / ORC ID: 0000-0002-3641-5801, CVU CONACYT ID: 40866

ID 1 ${ }^{\text {st }}$ Coauthor: Gabriela, Del Valle / ORC ID: 0000-0003-4559-5792, CVU CONACYT ID: 1000607

ID $2^{\text {nd }}$ Coauthor: Damián, Muciño-Cruz / ORC ID: 0000-0002-8710-9679, CVU CONACYT ID: 1000611

ID $3^{\text {rd }}$ Coauthor: Guadalupe, Hernandez-Morales / ORC ID: 0000-0002-6793-273X, CVU CONACYT ID: 74002

DOI: $10.35429 /$ EJDRC.2019.9.5.17.29

Received July 28, 2019; Accepted December 20, 2019

\begin{abstract}
In the children's movie The Incredibles there is a scene where Mr Incredible faces Bomb Voyage, while Incredi Boy wants to help Mr Incredible, Incredi Boy flies with Mr Incredible, who holds on to the hero's cloak, affecting Incredi Boy's flight plan. To understand how an oscillatory movement affects non-oscillatory movement, an experimental prototype was constructed with a particle of mass $\mathrm{m}$, attached to a rigid rod and without mass of length 1 , to a swivel of negligible mass, which was subject to a mass $M$. The swivel always remained on a horizontal plane, allowing the oscillatory movement of mass $\mathrm{m}$. Experimental results were obtained by means of wireless sensors which recorded the spatial coordinates of the mass $\mathrm{m}$. Using Lagrangian mechanics we obtained the equations of motion and expressed the possible first integrals of movement, when the movement of the mass $M$ was: linear uniform (ULM), uniformly accelerated, (UAM), uniform circular (UCM), accelerated circular (ACM) and forced circular (FCM). The dynamics were analyzed, the equations of movement obtained, they were solved numerically, and the experimental results were compared to theoretical and numerical results.
\end{abstract}

Mechanics, Simulation, No-inertial-Movement

\section{Resumen}

En una película infantil hay una escena, Mr Increíble enfrenta a Bomb Voyage, el niño Incredi Boy quiere ayudar a $\mathrm{Mr}$ Increible, Incredi Boy vuela con $\mathrm{Mr}$ incré́ble, quien se sostiene de la capa del héroe, afectando el plan de vuelo de Incredi Boy. Para entender cómo afecta un movimiento oscilatorio al movimiento no oscilatorio, se construye un prototipo experimental con una partícula de masa $\mathrm{m}$, unida a una varilla rígida y sin masa de longitud 1 , a una rótula de masa despreciable, la cual está sujeta a una masa $\mathrm{M}$. La rótula permanece en todo instante sobre un plano horizontal y permite el movimiento oscilatorio de la masa $\mathrm{m}$. Se obtienen resultados experimentales que son recabados por medio de sensores inalámbricos que permiten la obtención de coordenadas espaciales de la masa $\mathrm{m}$. Usando mecánica Lagrangiana se obtienen las ecuaciones de movimiento, expresamos las posibles integrales primeras de movimiento, cuando el movimiento de la masa $\mathrm{M}$ es: rectilíneo uniforme, (MRU), uniformemente acelerado, (MRUA) circular uniforme (MCU), circular acelerado (MCA) y circular forzado (MCF). Se analiza la dinámica, se obtienen las ecuaciones de movimiento, se resuelven numéricamente, y se contrastan los resultados experimentales con resultados teóricos y numéricos.

Mecánica, Simulación, Sistema-no-inercial

Citation: ESPINDOLA-HEREDIA, Rodolfo, DEL VALLE, Gabriela, MUCIÑO-CRUZ, Damián and HERNANDEZMORALES, Guadalupe. Oscillation of a pendulum subject to a horizontal trajectory with different kinds of motion. ECORFAN Journal-Democratic Republic of Congo. 2019, 5-9: 17-29

\footnotetext{
* Correspondence to Author (email: roeshe@ azc.uam.mx)

$\dagger$ Researcher contributing first author.
} 


\section{Introduction}

In the children's movie The Incredibles I, Mr. Incredible when facing one of the villains, the famous Bomb Voyage, is helped by his biggest fan, a boy named Buddy Pine, who would later become his archenemy. Mr. Incredible clarifies to the boy that he works alone and does not want to deal with Incrediboy (Buddy Pine), Incrediboy insists to be part of the incredible celebrities who help the world against the wicked and considers that searching for help can be a way to start participating with Mr. Incredible.

On his way out, the evil Bomb Voyage places one of his bombs on the boy's cape, Mr. Incredible upon realizing it, releases the villain and runs to rescue the child. The boy is equipped with shoes that allow him to fly, he starts his flight just when Mr. Incredible grabs his cape, the child's flight then becomes unstable and erratic due to the oscillation of the body of the super Hero with the boy's cape and, of course, to Mr. Incredible's own weight.

We take this fact to study the dynamics of a movement like that described in the movie. Our goal is to understand how a threedimensional (3D) movement for a given object is disturbed by the oscillation of a second object subject to the first. This kind of movements occur in real life and this research may have applications for practical cases, for example: when a helicopter by means of a cable, carries a certain mass, like a sculpture, or moves granular material to put out a fire, etc.

Carrying out the investigation for a 3D case of a flying object is our final goal; for this, we show results for certain cases of the study system, which will allow us to subsequently establish the general case in 3D.

The system (see Figure 1) is formed by a particle of mass $m$, which is attached through a rigid rod of length $l$ without mass to a ball of negligible mass, which is subject to another object of mass $M$; the swivel at all times remains on a horizontal plane and allows the oscillatory movement of the particle of mass $m$. The movement of the mass particle $M$ obeys the following cases: uniform linear motion (ULM), uniformly accelerated linear motion (UALM), uniform circular motion (UCM), uniformly accelerated circular motion (UACM).
The dynamic properties of the system are studied through Lagrangian dynamics, the energies are obtained to subsequently obtain the equations of motion through the EulerLagrange equations and thus study the coupling between the linear and angular variables.

Likewise, the study is carried out from an experimental device in accordance with the system described above, results are obtained on the acceleration of mass $M$ with the help of wireless communication by means of sensors, which are provided with accelerometer, compass and gyroscope, allowing the obtention of linear and angular acceleration values. Numerical results of the motion equations are also obtained by solving the system of differential motion equations for each case. We discuss their tendency to chaos and contrast the experimental results with the numerical ones.

\section{Theory}

a)

Uniform Linear Motions and Uniformly Accelerated Linear Motion

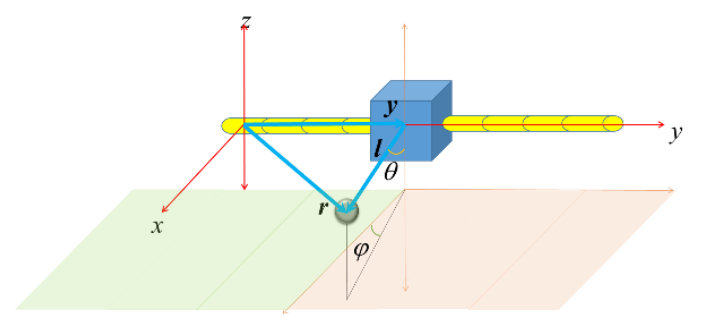

Figure 1 Description of the study system

We first consider the case when the mass $M$ moves in a straight line in the direction of the $y$-axis, the inertial reference system selected to study both the movement of the mass $M$ and the pendulum of mass $m$ and length $l$ is shown in Figure 1 and it is determined by the $x, y, z$ axes. Note that the position vectors: $\vec{r}$ that locates the mass $m, \vec{y}$ that locates the mass $M$ and $\vec{l}$ that locates the mass $m$, from the center of mass of $M$, form a triangular polygon, which allows the three vectors to be related:

$\vec{r}=\vec{y}+\vec{l}$

The $\vec{y}$ and $\vec{r}$ vectors are given by the following two equations:

$\vec{y}=y \hat{\jmath}$ 


$$
\begin{gathered}
\vec{r}=l \cos \varphi \sin \theta \hat{\imath}+ \\
l \sin \varphi \sin \theta \hat{\jmath}-l \sin \theta \hat{k}
\end{gathered}
$$

As the coordinate of interest is $\vec{r}$, in order to construct the kinetic energy of the pendular mass: $K=m \dot{r}^{2} / 2$, in the case of the pendulum, it is necessary to obtain $\vec{r}$; with (13 ) and then taking the internal product $\dot{r}^{2}=\dot{\vec{r}}$. $\dot{\vec{r}}$ to establish the kinetic energy of the mass $m$ :

$K_{m}=\frac{1}{2} m\left(l^{2} \sin \theta^{2} \dot{\theta}^{2}+(-l \sin \theta \sin \varphi \dot{\theta}+\right.$ $l \cos \theta \cos \varphi \dot{\varphi})^{2}+(\dot{\mathrm{y}}+l \cos \theta \sin \varphi \dot{\theta}+$ $\left.l \cos \varphi \sin \theta \dot{\varphi})^{2}\right)$

It is possible to observe that this kinetic energy depends on the variables: $(\dot{y}, \theta, \dot{\theta}, \varphi, \dot{\varphi})$. The kinetic energy of the mass object $M$ will be given by:

$$
K_{M}=\frac{1}{2} M \dot{y}^{2}
$$

Where $\dot{y}$ is the velocity of mass $M$. Because it travels on an equipotential gravitational surface, the change in its gravitational potential energy is zero and the contribution to gravitational potential energy is due only to the pendular mass, which is:

$$
U=-m g l \cos \theta
$$

The Lagrangian function (Herber Goldstein, 2000) of the system is obtained in the standard way: $L=K-U$. Through the EulerLagrange equation: $\frac{d}{d t} \frac{\partial L}{\partial \dot{q}}-\frac{\partial L}{\partial q}=0$, the three equations of motion are obtained, for the timedependent dynamic variables: $\theta(t), \varphi(t), y(t)$ as follows:

$$
\begin{gathered}
\ddot{\theta}=\frac{2 g \sin \theta+l \sin 2 \theta \dot{\theta}^{2}+2 l \sin 2 \varphi \dot{\theta} \dot{\varphi}+2 \cos \theta \sin \varphi \ddot{y}}{-2 l+l \cos 2 \theta+l \cos 2 \varphi} \\
\ddot{\varphi}=-\frac{\sec \varphi \sin \theta \ddot{\mathrm{y}}}{l}+\tan \varphi \dot{\theta}^{2}+\tan \varphi \dot{\varphi}^{2} \\
\ddot{\mathrm{y}}=\frac{l m \sin \theta \sin \varphi \dot{\theta}^{2}}{m+M}-\frac{2 l m \cos \theta \cos \varphi \dot{\theta} \dot{\varphi}}{m+M}- \\
\frac{l m \sin \theta \sin \varphi \dot{\varphi}^{2}}{m+M}-\frac{l m \cos \theta \sin \varphi \ddot{\theta}}{m+M}-\frac{l m \cos \varphi \sin \theta \ddot{\varphi}}{m+M}
\end{gathered}
$$

It is possible to continue along this path, so it is necessary that the set of three ordinary differential equations of the second order be reduced.
It is observed that the dynamic variables $\ddot{\theta}(t), \ddot{\varphi}(t), \ddot{y}(t)$ are coupled to reduce the system of equations, substitute (9) in equations (7) and (8) to obtain a set of two differential equations with two variables; with this, the information of the linear variable $y$ is lost, which can be known from the results for $\theta$ and $\varphi$.

However, it is possible to obtain more information because in this Lagrangian the $y$ coordinate is a cyclic coordinate, since there is no dependence on it in the Lagrangian and only depend on $\dot{y}$, that is:

$\frac{\partial L}{\partial \dot{y}}=p_{y}=M \dot{y}$

Where $p_{y}$ is the linear momentum of mass $M$, combining the results, we have:

$$
\dot{y}=-l(\cos \theta \sin \varphi \dot{\theta}+\cos \varphi \sin \theta \dot{\varphi})
$$

Equation (11) is integrated to obtain the following result:

$$
\begin{aligned}
& y=\int d y=-l \int(\cos \theta \sin \varphi d \theta)- \\
& -l \int(\cos \varphi \sin \theta d \varphi)= \\
& =-2 l \sin \theta \sin \varphi
\end{aligned}
$$

This result is interesting because although it is true it is negative, the function $\sin (x)$ is sometimes positive and sometimes negative; it is also assumed that although the variables $\theta$ and $\varphi$ are coupled, we do not know if they are found in phase or not, so that result indicates that certainly the car sometimes moves forward and sometimes backwards. With these results, we prepared the simulation to analyze the dynamic states of the system.

\section{b) Circular Motion \\ c)}

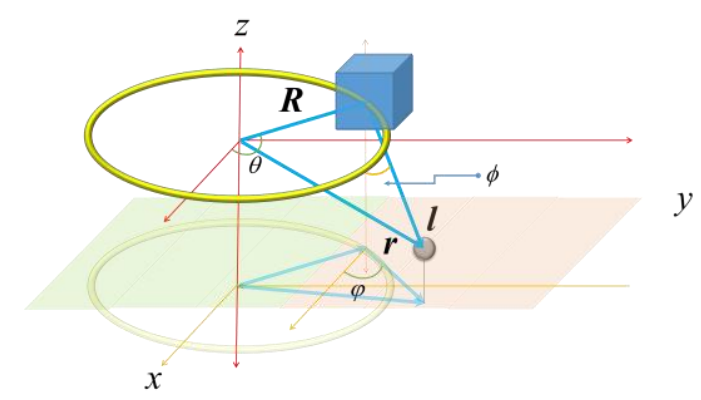

Figure 2 Description of the study system in uniform circular motion 
For this case, we considered Figure 2 and observed that the triangular polygon is formed by the vectors $\vec{R}, \vec{l}$ and $\vec{r}$. The way in which the three vectors are related is given by:

$\vec{r}=\vec{R}+\vec{l}$ is written as:

In rectangular coordinates the vector $\vec{R}$

$\vec{R}=R \cos \theta \hat{\imath}+R \sin \theta \hat{\jmath}$

Where $R$ is the radius of the circumference where the object of mass $M$ will move and $\theta$ is the azimuth angle. The vector $\vec{l}$ locates the pendular mass from the center of mass of the object of mass $M$, the pendular mass can move in three dimensions, so that the vector $\vec{l}$ is written based on the variables of Figure 2 as:

$$
\begin{gathered}
\vec{l}=l \sin \phi \cos \varphi \hat{\imath}+l \sin \phi \sin \varphi \hat{\jmath}- \\
l \cos \phi \hat{k}
\end{gathered}
$$

with (10) (11) and (12) $\vec{r}$ is established

as,

$$
\begin{aligned}
& \vec{r}=(R \cos \theta+l \sin \phi \cos \varphi) \hat{\imath}+ \\
& (R \sin \theta+l \sin \phi \sin \varphi) \hat{\jmath}-l \cos \phi \hat{k}
\end{aligned}
$$

by proceeding in the same way as for the linear case we have:

$$
\begin{aligned}
& K_{m}=\frac{1}{2} m\left(R^{2} \dot{\theta}^{2}+2 l R \dot{\theta}(-\cos \phi \sin (\theta-\right. \\
& \varphi) \dot{\phi}+\cos (\theta-\varphi) \sin \phi \dot{\varphi})+l^{2}\left(\dot{\phi}^{2}+\right. \\
& \left.\left.\sin \phi^{2} \dot{\varphi}^{2}\right)\right)
\end{aligned}
$$

and $K_{M}$ is obtained with:

$$
K_{M}=\frac{1}{2} M R^{2} \dot{\theta}^{2}
$$

The potential energy is only gravitational and is corresponding to the pendulum as follows:

$U_{g_{m}}=-m l g \cos \phi$

Of course, once the Lagrangian is obtained, we proceeded in the same way: through the equation of Euler Lagrange the equations of movement are obtained for the three dynamic variables:

$$
\begin{aligned}
& \ddot{\theta}=-\frac{l m \sin \phi \sin (\theta-\varphi) \dot{\phi}^{2}}{(m+M) R}- \\
& \frac{2 l m \cos \phi \cos (\theta-\varphi) \dot{\phi} \dot{\varphi}}{(m+M) R}-\frac{l m \sin \phi \sin (\theta-\varphi) \dot{\varphi}^{2}}{(m+M) R}+ \\
& \frac{l m \cos \phi \sin (\theta-\varphi) \ddot{\phi}}{(m+M) R}-\frac{l m \cos (\theta-\varphi) \sin \phi \ddot{\varphi}}{(m+M) R} \\
& \ddot{\phi}=-\frac{g \sin \phi}{l}+\frac{R \cos \phi \cos (\theta-\varphi) \dot{\theta}^{2}}{l}+ \\
& \cos \phi \sin \phi \dot{\varphi}^{2}+\frac{R \cos \phi \sin (\theta-\varphi) \ddot{\theta}}{l} \\
& \ddot{\varphi}=\frac{R \csc \phi \sin (\theta-\varphi) \dot{\theta}^{2}}{l}-2 \cot \phi \dot{\phi} \dot{\varphi}- \\
& \frac{R \cos (\theta-\varphi) \csc \phi \ddot{\theta}}{l}
\end{aligned}
$$

In this set of three second-order ordinary differential equations, the dynamic variables are also coupled. To solve the system, it is necessary that (21) and (22) are substituted in (20), to obtain an equation for $\ddot{\theta}$ which only depends on the generalized coordinates $\theta, \phi, \varphi, \dot{\theta}, \dot{\phi}$ and $\dot{\varphi}$. Then, once having that result for (20), it is also substituted in both (21) and (22) to obtain three equations dependent only on the generalized coordinates, and solve by numerical methods, the set of differential equations. Unlike the previous case, there is no cyclical coordinate here, which indicates that there is no conservation of any associated moment or amount of movement.

\section{Simulation}

In order to carry out the simulation of the described systems, a change of variable is usually suggested to reduce the system to a first order, the results obtained by the simulation correspond to the values $q$ and $\dot{q}$ since $\ddot{q}$ is known, which is described by equations (7-9) for the case of linear motion and (20-22) for circular motion.

The numerical method used to solve the differential equations is the Runge-Kutta method of order $4(R K 4)$. The choice of the method is essentially because it is the most standardized, we have it programmed and it has served for the numerical solution of other problems and dynamic systems that we have studied.

There are currently several computer programs that intrinsically have a variety of solution methods programmed for differential equations; however, we chose to develop our own code, because these programs represent a black box for the user.

ESPINDOLA-HEREDIA, Rodolfo, DEL VALLE, Gabriela, MUCIÑOCRUZ, Damián and HERNANDEZ-MORALES, Guadalupe. Oscillation of a pendulum subject to a horizontal trajectory with different kinds of motion. ECORFAN Journal-Democratic Republic of Congo. 2019 
This method is explained and applied for simple systems in books of numerical methods (Timothy Sauer, 2013), as well as in books of differential equations (W. E Boyce, 2004). The error due to the use of the method is of the order of the step size, which we denote by $\delta$ h raised to the 4 , that is, $\varepsilon r r=\Delta h^{4}$. The step size is defined as the partition of the integration interval $\delta h=(b-a) / n$, where $b$ is the upper limit of the interval, $a$ is the lower limit, $n$ the number of parts into which the interval is divided, which in fact corresponds to the number of iterations that will have to be done to cover the entire proposed integration interval (b-a). For case a) we have $\delta \mathrm{h}=0.0011$ and for case b) $\delta \mathrm{h}=0.0019$, throwing an error $\varepsilon r r=1.4 \times 10^{-12}, \varepsilon r r=1.4 \times 10^{-11}$, respectively.

\section{UALM simulation details}

Table 1 contains the values of the properties used in the simulation: $g$ is the value of the acceleration of gravity, equal to the value reported for Mexico City. The upper limit of the simulation was taken as 1.1 , because when exceeding this value, it has problems with the values obtained from the calculation, this is because in the equations to integrate, there are trigonometric functions such as tangent and reactive, which around this value is probable that the system approaches some asymptote, so the function is undetermined.

The length of the pendulum is denoted by $l, m$ is the pendular mass, and $M$ is the mass of the car. The rest are initial conditions (IC) to solve the equations. $N$ is the number of times we repeated the numerical experiment; each repetition of the experiment was performed with new ICs. In order to observe the changes produced by the new ICs, it was decided to only make small changes systematically, for this purpose changes were proposed only in the variables related to speed:

$\dot{y_{0}}=\dot{y}_{0}+0.09$
$\dot{\theta_{0}}=\dot{\theta_{0}}+0.09$
$\dot{\varphi_{0}}=\dot{\varphi}_{0}+0.10$

\begin{tabular}{|l|l|l|c|l|l|}
\hline \multicolumn{7}{|c|}{ UALM simulation } \\
\hline prop & value & unit & prop & value & unit \\
\hline $\boldsymbol{g}$ & 9.780 & $\mathrm{~m} / \mathrm{s}^{2}$ & $\boldsymbol{y}_{\mathbf{0}}$ & 0.1 & $\mathrm{~m}$ \\
\hline $\boldsymbol{a}$ & 0.000 & $\mathrm{adim}$ & $\dot{\boldsymbol{y}}_{\mathbf{0}}$ & 3.1 & $\mathrm{~m} / \mathrm{s}$ \\
\hline $\boldsymbol{b}$ & 1.100 & $\mathrm{adim}$ & $\boldsymbol{\theta}_{\mathbf{0}}$ & 1.1 & $\mathrm{grad}$ \\
\hline $\boldsymbol{n}$ & 1000 & $\mathrm{adim}$ & $\dot{\boldsymbol{\theta}}_{\mathbf{0}}$ & 5.3 & $\mathrm{rad} / \mathrm{s}$ \\
\hline $\boldsymbol{l}$ & 0.450 & $\mathrm{~m}$ & $\boldsymbol{\phi}_{\mathbf{0}}$ & 1.5 & $\mathrm{grad}$ \\
\hline $\boldsymbol{m}$ & 0.270 & $\mathrm{Kg}$ & $\dot{\boldsymbol{\phi}}_{\mathbf{0}}$ & 5.1 & $\mathrm{rad} / \mathrm{s}$ \\
\hline $\boldsymbol{M}$ & 0.332 & $\mathrm{~kg}$ & $\mathbf{N}$ & 21 & $\mathrm{adim}$ \\
\hline
\end{tabular}

Table 1 Initial property and condition values

\section{UACM Simulation Details}

In the case of the UACM simulation, the gravity acceleration value was taken in the same way as in the previous case, which is why it does not appear in Table 2.

\begin{tabular}{|l|l|l|c|l|l|}
\hline \multicolumn{7}{|c|}{ UACM simulation } \\
\hline prop & value & unit & Prop & value & unit \\
\hline $\boldsymbol{a}$ & 0.000 & $\mathrm{~m} / \mathrm{s}^{2}$ & $\boldsymbol{\theta}_{\mathbf{0}}$ & 1.1 & $\mathrm{~m}$ \\
\hline $\boldsymbol{b}$ & 1.100 & $\mathrm{adim}$ & $\dot{\boldsymbol{\theta}}_{\mathbf{0}}$ & 3.3 & $\mathrm{~m} / \mathrm{s}$ \\
\hline $\boldsymbol{n}$ & 7000 & $\mathrm{adim}$ & $\boldsymbol{\phi}_{\mathbf{0}}$ & 1.1 & $\mathrm{grad}$ \\
\hline $\boldsymbol{R}$ & 0.450 & $\mathrm{M}$ & $\dot{\boldsymbol{\phi}}_{\mathbf{0}}$ & 6.3 & $\mathrm{rad} / \mathrm{s}$ \\
\hline $\boldsymbol{l}$ & 0.350 & $\mathrm{M}$ & $\boldsymbol{\varphi}_{\mathbf{0}}$ & 1.1 & $\mathrm{grad}$ \\
\hline $\boldsymbol{m}$ & 0.270 & $\mathrm{Kg}$ & $\dot{\boldsymbol{\varphi}}_{\mathbf{0}}$ & 10.1 & $\mathrm{rad} / \mathrm{s}$ \\
\hline $\boldsymbol{M}$ & 0.332 & $\mathrm{Kg}$ & $\mathbf{N}$ & 25 & $\mathrm{adim}$ \\
\hline
\end{tabular}

Table 2 Initial property and condition values

Table 2 shows the values of the parameters used in the UACM simulation, as well as the required $\mathrm{ICs}$, to obtain their solution. The parameter $R$ appears, which represents the radius of the circle on which the car rotates. The ICs are for the angles, being $\theta$ the polar angle, $\phi$ the zenith angle, and $\varphi$ the azimuth angle. Likewise, the ICs were changed for 25 numerical experiments, the following rules being in this case:

$\dot{\theta_{0}}=\dot{\theta_{0}}+0.09$

$\dot{\phi_{0}}=\dot{\phi}_{0}+0.10$

$\dot{\varphi_{0}}=\dot{\varphi}_{0}+0.10$

\section{Experimental development}

The experiment was carried out using a lowfriction car PASCO (ME-1240) and two rails were implemented. At the beginning, two rails that rest on two rigid rods were used, each wheel of the low friction car moves over each one, a "ball joint" (swivel) was implemented so that the pendulum moved freely, the pendular mass is a Naylamid sphere. 
To attach the pendulum to the lowfriction car, three parts were designed in Autodesk Inventor: one that serves as a special base to attach the swivel to the carriage, another to attach it to the pendulum rod and another to attach the rod to the pendulum mass. These pieces were 3D printed, an Anet A8 printer was used with Utimaker Cura 3.2.1.

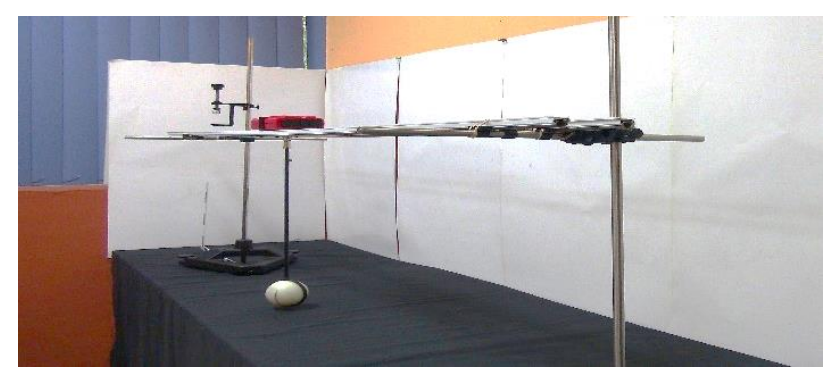

Figure 3 Experimental device for uniform linear motion

Likewise, special bases were designed to mount the rails on horizontal rods supported to a universal support. Through a video shot, the experiment was recorded for the two cases presented a) linear motion and b) circular motion, with a radius of curvature $R=1.5 \mathrm{~m}$. To follow the trajectory of the mobiles: car and pendulum, Traker Video Analysis and Modeling Tool were used, which allowed to collect the data of some of the studied properties, simultaneously data of the displacement of the low-friction car were collected by means of sensors with wireless "bluetooth" using as interface a cell phone in which the SPARKvue application was installed.

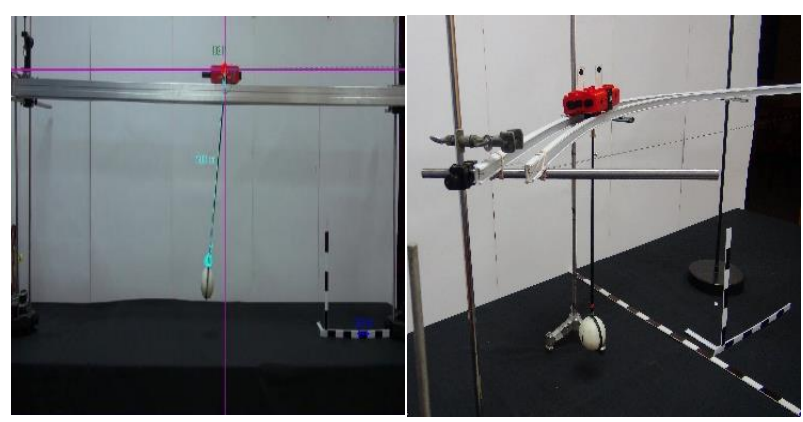

Figure 4 Experimental devices for both motions: uniform linear motion and uniform circular motion

To ensure that the initial car speed is the same in each video shot, a trigger was applied to the low-friction car (a piston activated by pressing a pivot on the top of the car, providing a boost, in this way it was also guaranteed that said impulse was of similar magnitude in each repetition of the experiment).
While the trigger was activated, the pendulum was placed at a certain angle, so that just at the moment when the car started its movement with a certain initial speed, the pendulum also began its wave motion.

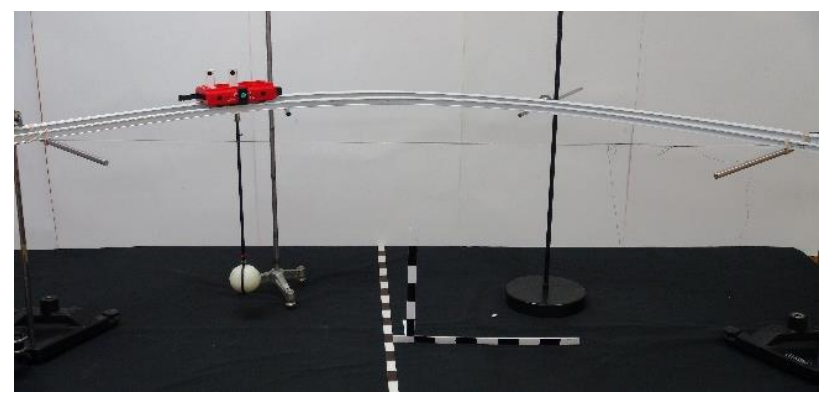

Figure 5 Experimental device for uniform circular motion

It is important to note that the video shots only allow us to analyze two dimensions, to monitor the movement of the pendulum in three dimensions requires preparing the scene much better and having more cameras and some other electronic devices to properly and correctly measure the dynamic variables of interest.

\section{Numerical Results}

\section{UALM}

The results obtained by simulation are several, because for each trial we get: time, position, velocity and acceleration of the object of mass $M$, as well as angular position, velocity and acceleration for the pendular mass $m$, given that the value of the pendulum length is known, it is possible to obtain the rectangular positions of the pendular mass $m$.

The phase diagrams show the behavior of the dynamic variables, these are of interest because the temporal parameter is eliminated and allow the observation of the existing changes between position and speed for each dynamic variable. Graph 1 shows the phase diagram for the $y$ coordinate, the solution for $y$ and $\dot{y}$, are given by (11-12), they certainly do not represent the numerical solution of the differential equation for $y$, but it is known from the numerical solutions for the angular coordinates $\theta$ and $\varphi$. In this graph, it is observed that the trajectory in the phase plane does not represent a closed curve, the change of ICs is also appreciated, by the different colors plotted, which shows the different trajectories that are quite similar to each other. 
It is not observed that the $y$ variable is sensitive to the change in ICs.

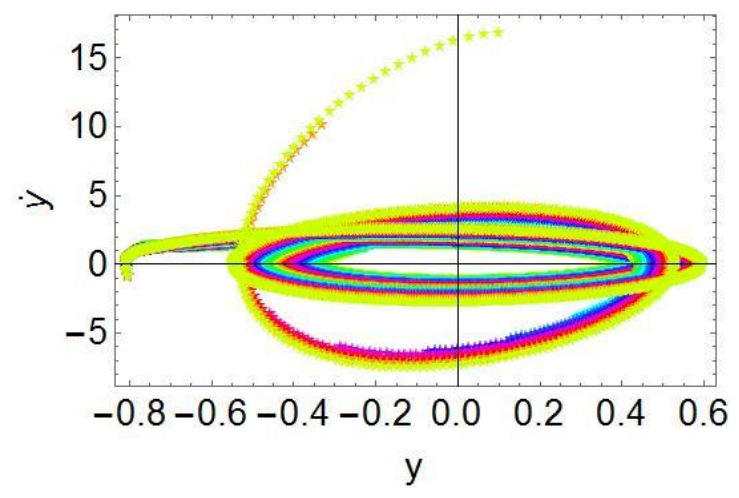

Graph 1 Phase diagram for coordinate $y$

Graph 2 shows the phase diagram for the angular coordinate $\theta$. In this diagram the result each trial within the simulation is welldefined, this result corresponds to the numerical solution of equations (7). Again, it is observed that all curves describe a similar shape. However, changes in ICs result in separate curves, enlarged or contracted, which shows signs that the $\theta$ variable is chaotic.

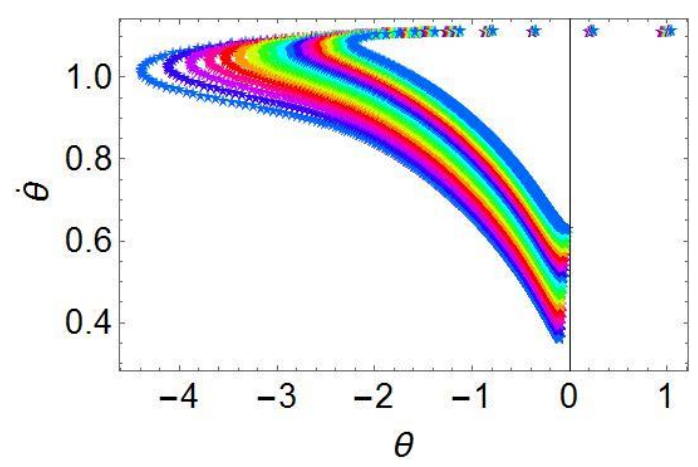

Graph 2 Phase diagram for coordinate $\theta$

Graph 3 presents the phase diagram corresponding to the angular variable $\varphi$. Of the three phase diagrams, this has the least structure, since the behavior between the variables $(\varphi$ vs. $\dot{\varphi})$ seems to be approximately logarithmic, the sensitivity to the changes of ICs shows only a shift in the curve.

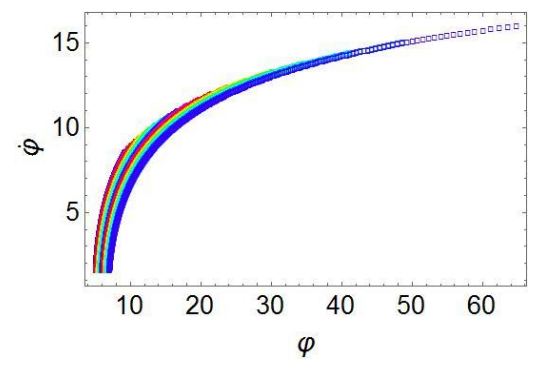

Graph 3 Phase diagram for coordinate $\varphi$
The result corresponding to the acceleration behavior in the direction $y$ and the direction of movement of the mass object $M$ proved to be relevant.

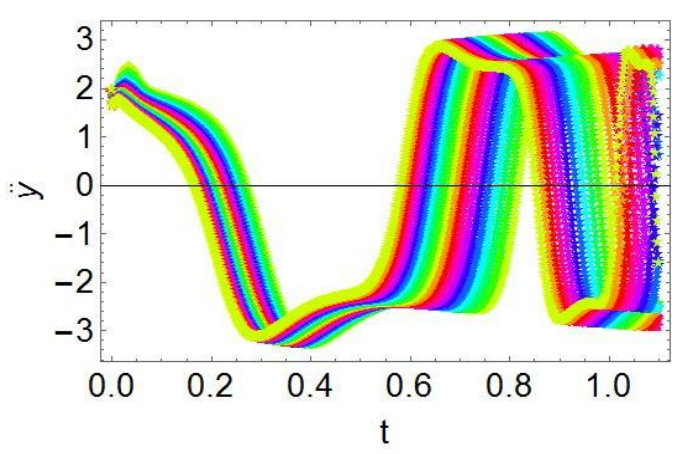

Graph 4 Result for acceleration in direction $y:(t v s . \ddot{y})$

Graph 4 shows the result for the 21 simulations performed. Acceleration is presented as a function of time, it exhibits greater sensitivity to changes in ICs; it is observed that the more the system moves from the origin coordinates, there is more variation between the trajectories, so there are more significant changes between each trial, which suggests that the system turns out to be chaotic. In this paper, no parameters related to the chaos of the system were measured, however, Graph 4, together with Graphs (2-3) motivates the possibility of doing so and directing attention in this direction. Another situation that can be seen in Graph 4 is that for some time intervals, the acceleration is negative, for others it is positive. The plot of the graph seems to tend approximately to an oscillatory movement with a certain frequency. Given the impossibility of measuring beyond the proposed values, it is not possible to observe this tendency with greater clarity, however, if it was possible to observe experimentally, the object of mass $M$ sometimes moves forward and sometimes backwards, showing a behavior similar to that in Graph 4.

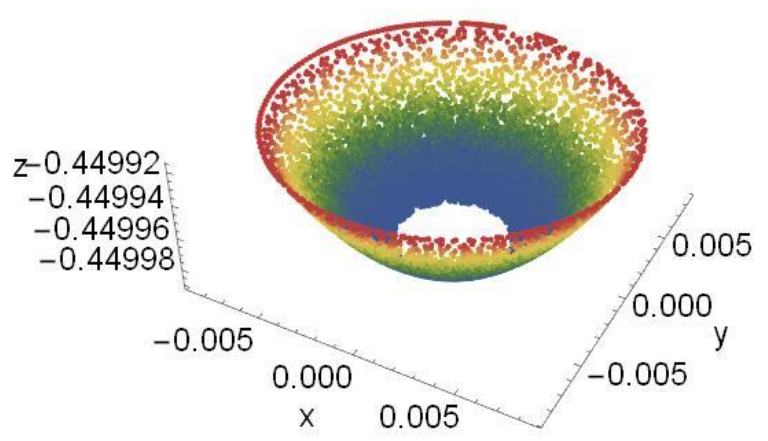

Graph 5 Result for the position of the pendular mass, in rectangular coordinates, from a fixed observer on object M.

ESPINDOLA-HEREDIA, Rodolfo, DEL VALLE, Gabriela, MUCIÑOCRUZ, Damián and HERNANDEZ-MORALES, Guadalupe. Oscillation of a pendulum subject to a horizontal trajectory with different kinds of motion. ECORFAN Journal-Democratic Republic of Congo. 2019 
Given the angular values obtained and the pendulum length, it is possible to obtain the position $(x, y, z)$ of the pendular mass.

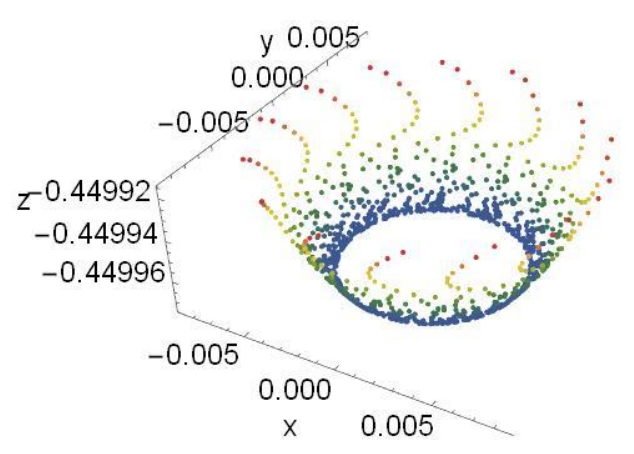

Graph 6 Result for the position of the pendular mass, in rectangular coordinates, for iteration 20. From a fixed observer on object $M$.

Graph 5 shows the trajectories for each of the 21 simulations performed, the result is that the positions of the pendular mass are located on the internal surface of a hemisphere of radius $l$, the gap that is observed at the bottom of the hemisphere result from the fact that the ICs do not start from zero. However, for any performed iteration, the pendulum will always be at a point on the surface of this hemisphere. The result shown in Graph 6 shows the iteration corresponding to trial number 20 , presenting greater clarity in this regard.

It is necessary to note that what is shown in Graphs 5 and 6 is the observation of the movement of the pendulum from an observer who is on the object of mass $M$. As we have previously mentioned, object $M$ moves with variable acceleration, therefore, this reference system corresponds to a non-inertial reference system. To have a result whose observation is from an inertial reference system, the pendulum's displacement must be measured from the fixed origin outside the object $M$, that is, the inertial reference zero, which is fixed to the left of the objects (see figure 1).

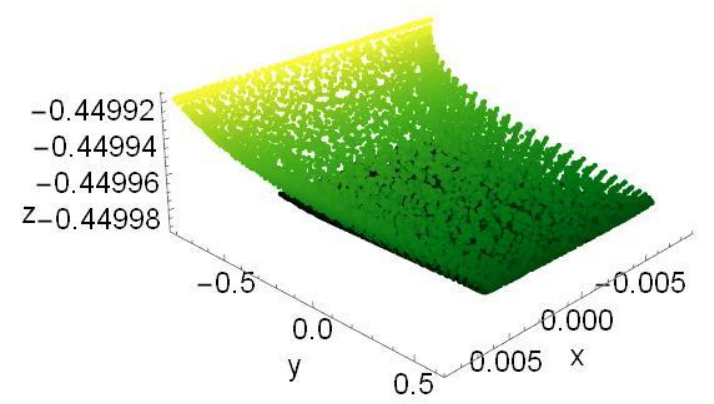

Graph 7 Result for the position of the pendular mass, in rectangular coordinates. From a fixed observer at the origin of coordinates
The observation from this inertial reference system is shown in figure 7.

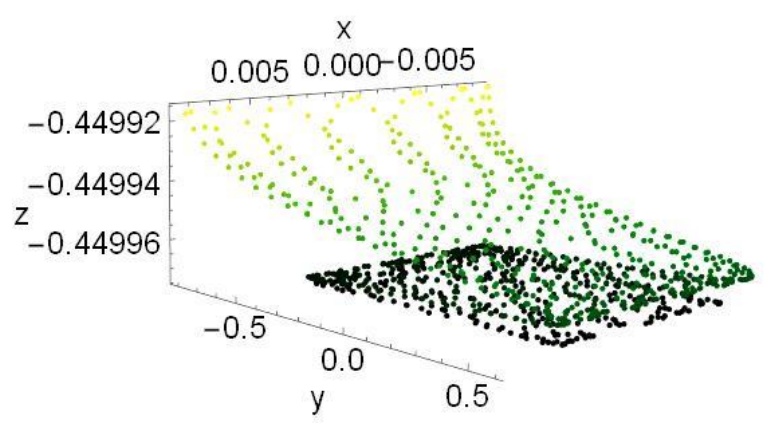

Graph 8 Result for the position of the pendular mass, in rectangular coordinates. From a fixed observer at the origin of coordinates

And the trajectory corresponding to trial 20 is shown in Graph 11, in both Graphs (7 and 8) the behavior of the coordinate $y$ can be observed, which shows the effect of the oscillatory movement in the direction in which the object of mass $M$ moves, displaying the behavior of a bending surface. These graphs have been presented as Graphs (5 and 6) exhibit the behavior of a spherical pendulum, which corresponds to the description of an observer looking at the fixed pendulum in $M$. However, a fixed observer in the inertial reference system $O$ will see what is shown in Graphs (7 and 8).

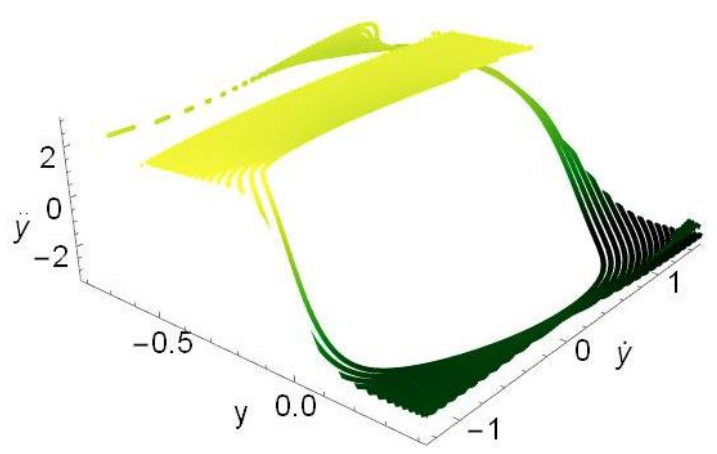

Graph 9 Coordinate $y$ behavior, the relationship between position, speed and acceleration is shown

Once the results are obtained for all the variables, it is possible to review the behavior of the three coordinates simultaneously, this behavior is presented for the variables $y$ and $\varphi$ in Graphs (9 and 10). 


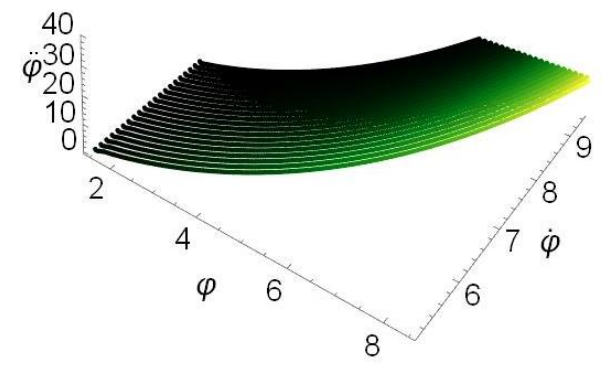

Graph 10 Behavior of the angular coordinate $\varphi$, the relationship between position, velocity and angular acceleration is shown. The behavior of the angular coordinate $\varphi$ is quite stable, it presents only a small slope.

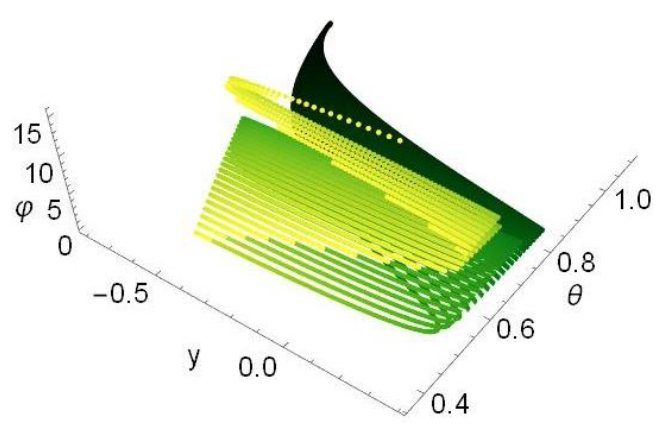

Graph 11 Behavior between both linear and angular positions. It can be observed a behavior that essentially has the same origin, but which, depending on the CIs, concludes at another point, that is, it shows a chaotic behavior mainly for the coordinate $\theta$.

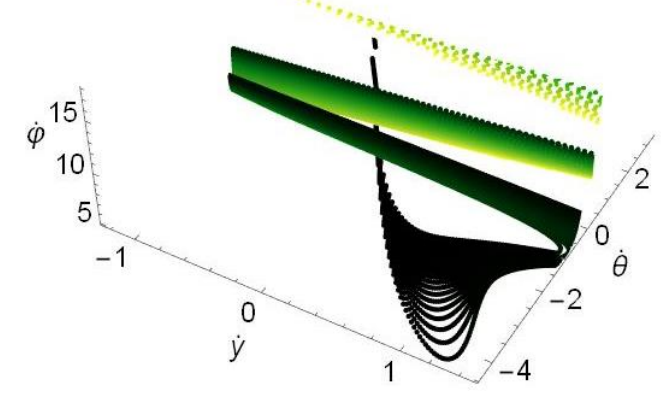

Graph 12 Behavior for linear and angular speeds. It is observed again that the graphs have a common origin, however the greatest change is provided by the $\theta$. coordinate.

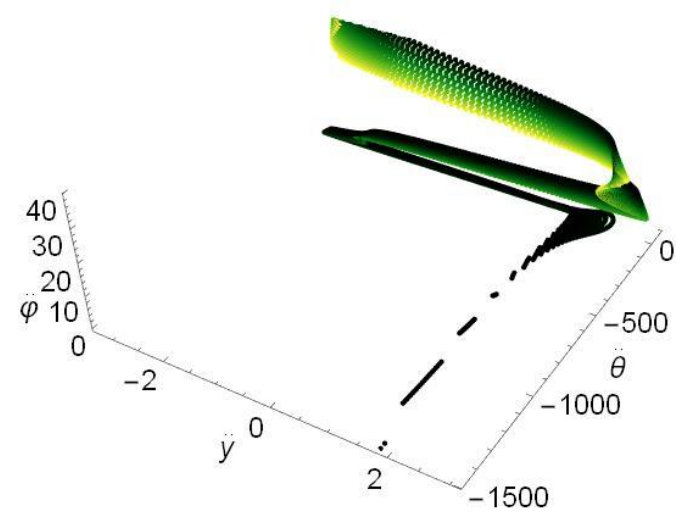

Graph 13 Acceleration behavior, a clear tendency to chaos in the acceleration behavior is observed.
Graphs (11-13) show the coupling behavior of the dynamic variables of the blockpendulum system and provide information on the effect of the linear movement of the mass block $M$ by effect of or as a consequence of an oscillatory movement of the mass pendular $m$.

\section{UACM}

The numerical results for the case of circular motion allow us to obtain results on the dynamic variables $\theta, \phi$ and $\varphi$, and on their temporal derivatives with which the phase diagrams can be constructed, as well as to observe the temporal evolution of each variable. Graph 14 presents the phase plane of the polar angular variable $\theta$, which is the car's direction. A simple comparison between Graphs 1 and 14, allows us to observe that the behavior is different, due to the trajectories that the movement associated with the object of mass $M$ follows.

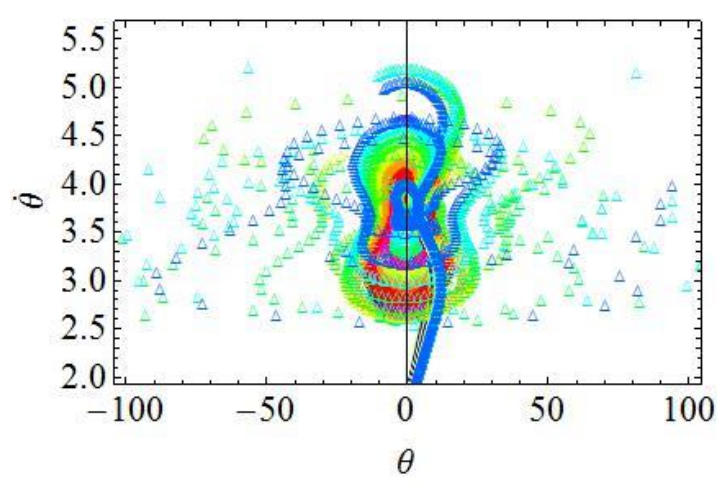

Graph 14 Phase plane for coordinate $\theta$

However, in both cases the curves are open, it is also observed that for both cases the paths of the same trial cross more than once.

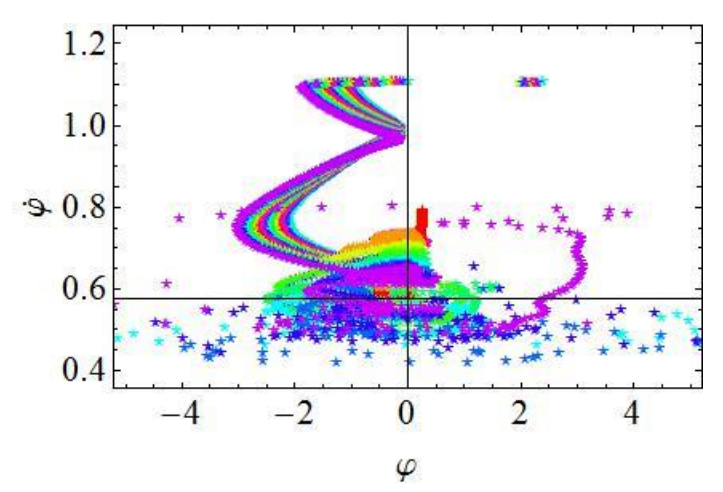

Graph 15 Phase diagram for the angular variable $\square$

A much more chaotic result is appreciated for the UACM than for the UALM. 


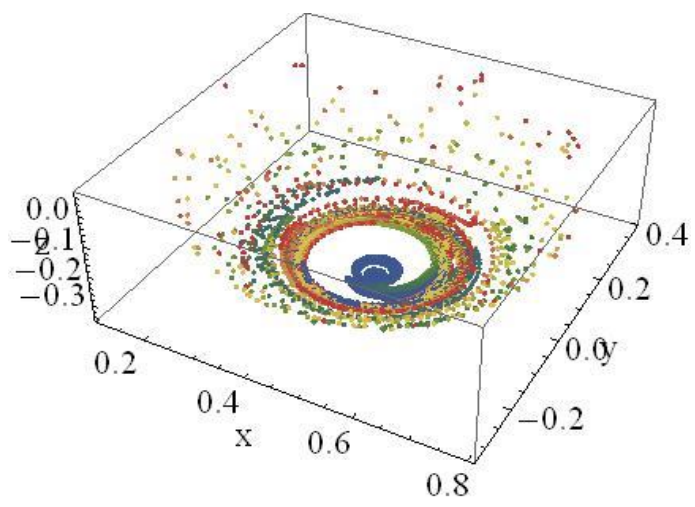

Graph 16 Space trajectories for the behavior of pendular mass in uniformly accelerated circular motion

Graph 15 shows the phase diagram for the azimuth angle $\varphi$, a comparison between Graphs 2 and 15 shows that in the case of the UACM, the azimuth coordinate is more chaotic. Similarly, it is possible to obtain the $x, y$ and $z$ coordinates of the pendular mass $m$, shown in Graph 16, where the spatial trajectory is observed for the 21 simulations performed for this case. The comparison between Graphs 5, 16 and 7, show a different behavior, a tendency to chaos. The movement of the pendular mass is erratic, even though at the beginning the internal structure of the trajectories presents a systematic and similar behavior to that of a spiral.

A two-dimensional view is presented in Graph 17. Different tests were made proposing various changes for the ICs; it was observed that the change for the angular velocity $\dot{\theta}$ presents an offset of the center of the circle for the initial spiral paths, the end values for each trajectory are very scattered, so the behavior shown by the pendular mass also tells us that in the circular motion of the object of mass $M$, its behavior is erratic.

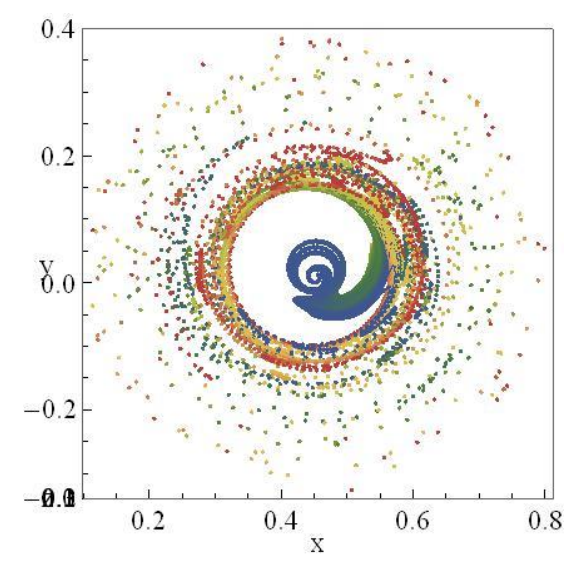

Graph 17 Spatial trajectories for the behavior of the pendular mass in the uniformly accelerated circular motion in two dimensions, corresponding to the coordinates $(\mathrm{x}, \mathrm{y})$.

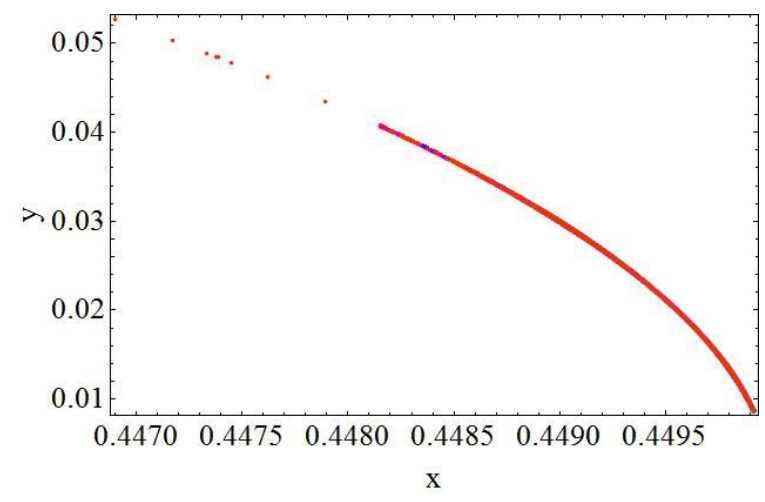

Graph 17 Spatial trajectories for the behavior of the pendular mass in the uniformly accelerated circular motion in two dimensions, corresponding to the coordinates $(\mathrm{x}, \mathrm{y})$

Finally, Graph 18 shows the result for the trajectory of the object of mass $M$. Since the entire interval was not integrated, the trajectory shows only one segment; the total of the 21 results of the simulations fall on the same curve, which was expected.

\section{Experimental Results}

\section{UALM}

The experimental results obtained allow us to make purely qualitative comparisons. In order to obtain precise results to quantitatively contrast with the numerical results, we are solving the experimental inconveniences.

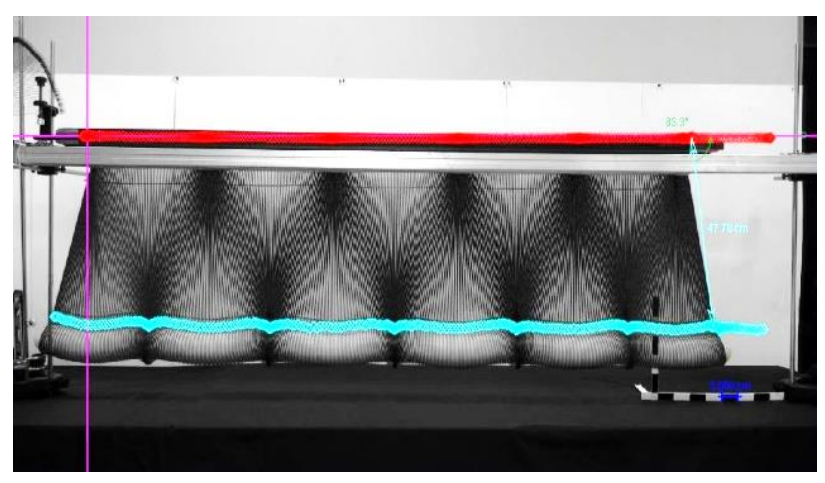

Figure 6 Space trajectories for pendular mass behavior in accelerated linear motion

Figure 6 shows a frame of the analysis by means of Tracker, the red curve corresponds to the tracking done to the object of mass $M$, while the blue curve represents the monitoring of the pendular mass $m$; in the same frame the movement of the rod is recorded. The effect of the pendulum is observed when moving inside the hemisphere, as indicated by Graphs (5-6). 


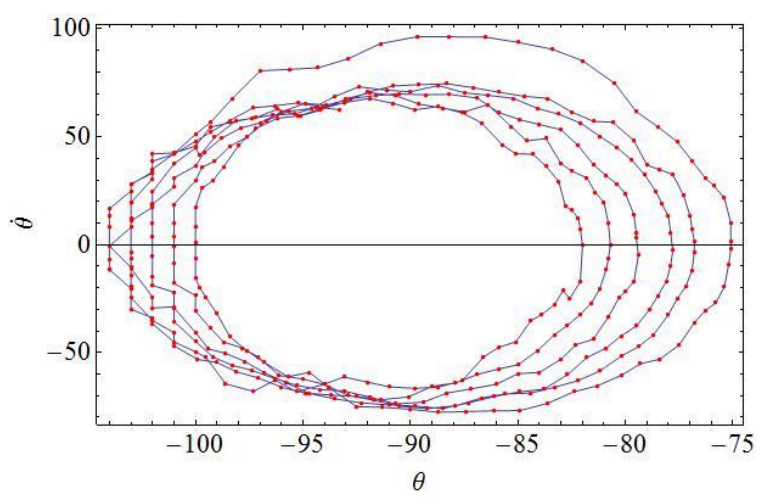

Graph 19 Trajectories corresponding to positions $(\theta, \dot{\theta})$ of the pendular mass $m$.

Following the pendular mass is complicated through Tracker, since the video only allows us to observe two dimensions. The results are affected both by the perspective, as well as the depth of the shot, also by the focus, so they contain a considerable experimental error. Graph 19 shows the phase plane of the variable $\theta$, the curve is comparable to that shown in Graph 6 in two dimensions, showing a qualitative agreement between them.

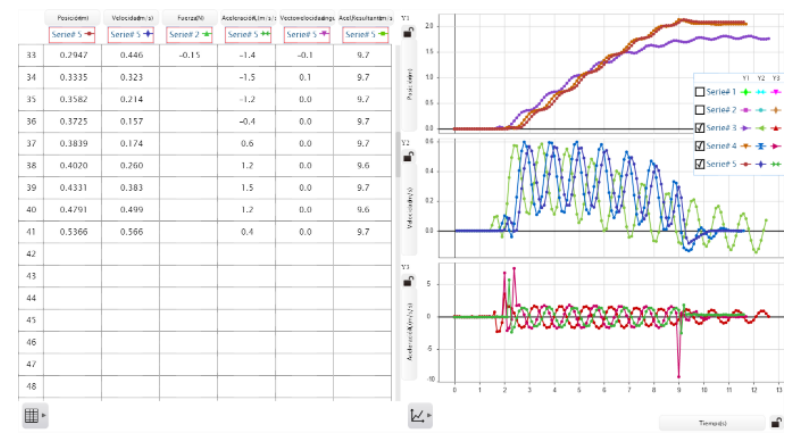

Graph 20 Results obtained by means of the sensor for linear motion

Graph 20 shows the results obtained by means of the sensor placed in the object of mass $M$. The first graph offers results for the position, observing the oscillations for three different experiments, these oscillations are also present in the graph corresponding to the simulation result. They differ in the trend of the slope. The second graph corresponds to that of the velocity in $y$, which is also comparable with the numerical result. The third graph is for acceleration, showing the same effect as in Graph 4, where some values are positive and negative.

\section{UACM}

The results corresponding to the circular motion are presented in Figure 7 , where, again, the monitoring on the object of mass $M$ moving on the rail forced to follow a circular motion is observed. The trajectory is drawn in red, while the monitoring of the pendular mass $m$ is in blue, the monitoring of the rod is also shown.

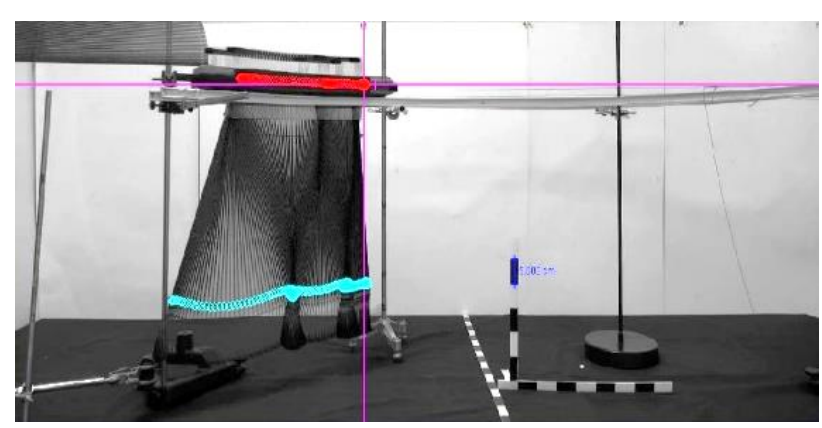

Figure 7 Trajectories corresponding to the positions of the pendular mass $\mathrm{m}$ and the object $\mathrm{M}$ in the circular motion

Figure 7 shows a qualitative agreement with the numerical result. To be clear on this, it is necessary to observe Graph 21, which shows the spiral behavior also shown by Graph 16 .

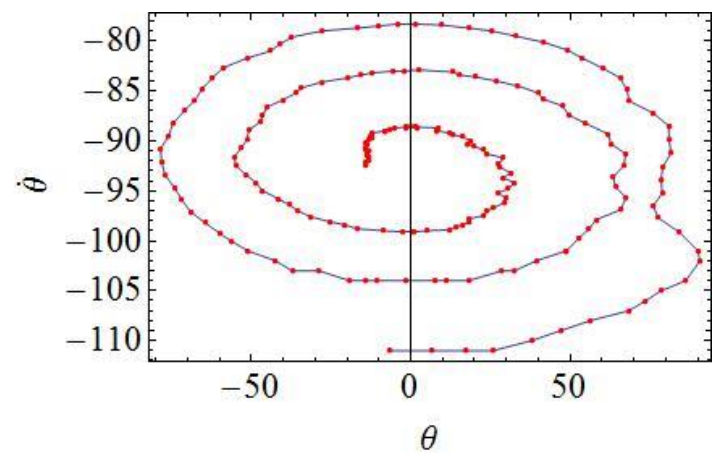

Graph 21 Results obtained by means of the sensor for linear motion

Finally, Graph 22 shows the results obtained by the sensor for the movement of the car of mass $M$.

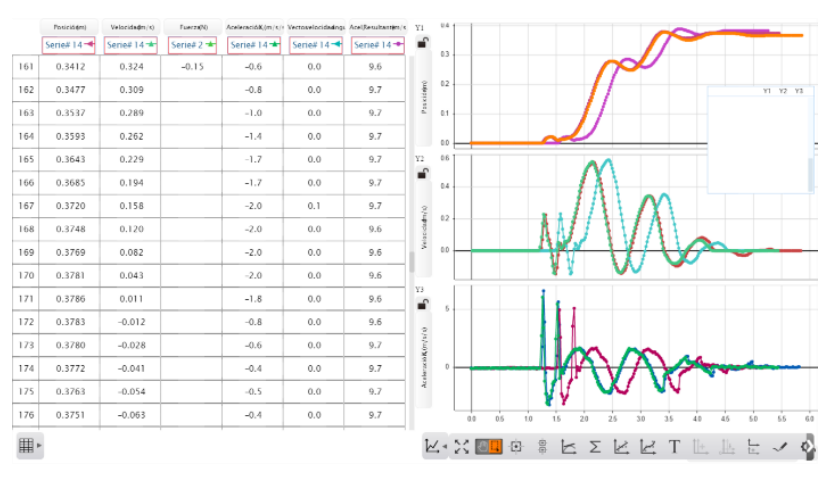

Graph 22 Results obtained by means of the sensor for circular motion 


\section{Conclusions}

We have presented the theoretical analysis of the system composed of an object of mass $M$ to which a pendulum of length $l$ and pendular mass $m$ was attached, moving in two kinds of movements: uniformly accelerated linear and uniformly accelerated circular motion. At first, we assumed that both movements were uniform; however, the analysis showed that due to the coupling of the objects and the oscillation of the pendulum the movement of the object of mass $M$ was affected, so that its acceleration did not turn out to be uniform.

Theoretical analysis showed that despite being a system with variable acceleration, the linear momentum of the object of mass $M$ was preserved, but not that of the pendular mass.

A set of numerical simulations was developed to obtain the solutions of the differential equations.

From the analysis of the numerical results, it is concluded that the system, both in linear and circular motion, is chaotic; thus, posing the possibility of studying them through the methodology used to analyze chaos, such as: Poincaré sections and Lyapunov coefficients, for example. In this way, it is possible to offer a more precise quantitative evaluation of the chaotic behavior of the system.

The corresponding experimental prototypes were developed to carry out a series of experiments and gather information with the use of proprietary tools such as Tracker. Likewise, wireless devices were used to phenomenologically verify both numerical and theoretical results. Because the experimental part requires even more care and that the pendulum movement occurs in $3 \mathrm{D}$, a qualitative agreement was found in the comparisons made for the numerical and experimental results. However, we concluded that it is necessary to improve the obtention of the experimental results so that the comparison between the two is quantitative and not only qualitative.

The study of this kind of systems is important since they are commonly used, for example, in the handling of cranes that carry loads, airplanes and/or helicopters that carry buoys to put out fires.
The object transported behaves like the pendular mass and its oscillatory movement affects the movement of the transporting object. The analysis shown so far has allowed us to reach theoretical solutions, which enable us to understand the behavior of the system; along with numerical solutions that help to understand its tendency to chaos and also contribute to improve the use of its implementations; and experimental solutions that allow us to generate more adequate prototypes for the required purposes.

\section{References.}

Douglas Brown. (2013). Sharing video experiments with tracker digital libraries. May 15, 2019, from Tracker Website: https://physlets.org/tracker/

Enrique, C., Yanitelli, M., y Giorgi, S. (2019). Perfiles conceptuales como instrumentos de evaluación de una intervención didáctica. Avances en la enseñanza de la Física, 1(1), 7399.

Herbert Goldstein, Charles Poole, John Safko. (2000). Classical Mechanics. New York: Addison Wesley.

Jaime De la Colina y Jesús Valdés. (2010). Péndulo de prueba para el estudio dinámico de modelos estructurales. Revista de Ingeniería Sísmica, No 82, pp 35-56. May 17, 2019, from Scielo Data base.

Jorge V. José and Eugene J. Saletan. (1998). Classical Dynamics. England: Cambridge University Press.

Lorena A. Lemus C. (2019). Simulación computacional, desarrollo teórico y comparación experimental de un péndulo elástico. Universidad de San Carlos de Guatemala Escuela de Ciencias Físicas y Matemáticas, Physics department: Bachelor thesis in Applied Physics.

Timothy Sauer. (2013). Numerical Methods. Chicago: PEARSON.

W. E. Boyce and R. C. Di Prima. (2004). Elementary Differential Equations and Boundary Value Problems. New York: Wiley. Wolfram Research, Inc., Mathematica, Version 11.3, Champaign, IL (2018) 
Zúñiga, Francisco Agustín; Morales, Edgar Javier (2017). Diseño de una secuencia didáctica para el aprendizaje de la pendiente como razón de cambio para alumnos de nivel medio superior utilizando herramientas tecnológicas. In Serna, Luis Arturo (Ed.), Acta Latinoamericana de Matemática Educativa (pp. 1495-1504). Mexico, Mexico City: Comité Latinoamericano de Matemática Educativa. 